\title{
Development of a novel method for the quantification of tyrosine 39 phosphorylated alpha- synuclein in human cerebrospinal fluid
}

Chan Hyun Na ( $\square$ chanhyun@jhmi.edu )

Johns Hopkins University https://orcid.org/0000-0002-3622-2938

Gajanan Sathe

National Institute of Mental Health and Neuro Sciences

Liana S. Rosenthal

Johns Hopkins University School of Medicine

Abhay R. Moghekar

Johns Hopkins University School of Medicine

Valina L. Dawson

Johns Hopkins University School of Medicine

Ted M. Dawson

Johns Hopkins University School of Medicine

Akhilesh Pandey

Mayo Clinic Minnesota

\section{Research}

Keywords: Parkinson's disease, \-synuclein, phosphotyrosine, cerebrospinal fluid, parallel reaction monitoring

Posted Date: February 28th, 2020

DOI: https://doi.org/10.21203/rs.2.20088/v2

License: (a) (1) This work is licensed under a Creative Commons Attribution 4.0 International License.

Read Full License

Version of Record: A version of this preprint was published at Clinical Proteomics on May 4th, 2020. See the published version at https://doi.org/10.1186/s12014-020-09277-8. 


\section{Abstract}

Background: Parkinson's disease (PD) is the second most prevalent neurodegenerative disorder. Biomarkers that can help monitor the progression of PD or response to disease-modifying agents will be invaluable in making appropriate therapeutic decisions. Further, biomarkers that could be used to distinguish PD from other related disorders with PD-like symptoms will be useful for accurate diagnosis and treatment. C-Abl tyrosine kinase is activated in PD resulting in increased phosphorylation of the tyrosine residue at position 39 of a-synuclein (a-syn) (pY39 a-syn), which contributes to the death of dopaminergic neurons. Because pY39a-syn may be pathogenic, monitoring pY39 a-syn could allow us to diagnose presymptomatic PD and help monitor disease progression and response to treatment. We sought to investigate if increased phosphorylation of pY39 a-syn can be detected in the CSF of PD patients by targeted mass spectrometry. Methods: Here, we report a two-step enrichment method in which phosphotyrosine peptides including pY39 a-syn containing peptide were first enriched with an antiphosphotyrosine antibody followed by a second round of enrichment by titanium oxide (TiO 2 ) beads. Accurate quantification was achieved by the addition of a synthetic heavy version of pY39 a-syn peptide added before enzymatic digestion. Results: Using the developed enrichment methods and optimized parallel reaction monitoring assays, we detected pY39 a-syn peptide in human CSF and demonstrate that the ratio of pY39 a-syn to total a-syn was significantly increased in the CSF of patients with PD. Conclusions: We anticipate that this optimized two-step enrichment-based PRM detection method will help monitor c-Abl activation in PD patients and can also be used to quantify other phosphotyrosine peptides of low abundance in biological samples.

\section{Introduction}

Although the exact pathogenic mechanism of PD has yet to be established, a-synuclein (a-syn) is an important mediator and moreover the phosphorylation of a-syn may contribute to the pathogenesis via increased aggregation and toxicity [1, 2]. Recently, tyrosine at position 39 of a-syn ( pY39 a-syn) has been shown to be closely correlated to disease severity and progression $[1,3,4]$. Thus, pY39 a-syn levels in the brain could potentially serve as a marker for presymptomatic diagnosis, disease progression, and therapeutic response. Since pY39 a-syn is increased in the brain of PD patients, we postulated that this increased phosphorylation could be reflected in the CSF [5].

For targeted detection and quantitation of known proteins or post-translational modifications (PTMs) on those proteins, parallel reaction monitoring (PRM) mass spectrometry has been widely used $[6,7]$. When the abundance of a target protein is too low, the target protein or the derived peptide sometimes can be detected using various enrichment methods such as immunoprecipitation or affinity purification of target proteins or peptides [8-10]. Here, we report a method specifically developed to detect pY39 a-syn peptide in human CSF combining an enrichment method with the PRM mass spectrometry approach. Because the abundance of pY39 a-syn is too low to be detected even with a conventional enrichment method, we developed a two-step enrichment approach using an anti-phosphotyrosine antibody and $\mathrm{TiO}_{2}$ beads followed by PRM analysis for detection and quantitation of pY39 a-syn. This method allowed us to 
establish that the ratio of pY39 a-syn to total a-syn in the CSF can serve as a potential biomarker for the diagnosis and prognosis of PD. Furthermore, this method is applicable to the extremely sensitive detection of other phosphotyrosine peptides as well.

\section{Methods}

\section{Collection of CSF samples}

The CSF specimens were collected from normal pressure hydrocephalus (NPH) patients, PD patients or cognitively normal healthy control individuals evaluated by investigators at the Johns Hopkins Hospital. The CSF samples from NPH patients were used for method optimization. The CSF samples from PD or control individuals were used to compare pY39 a-syn peptide levels between the two groups. The individuals who are cognitively normal or show PD symptoms were diagnosed after extensive clinical and cognitive testing. All PD patients met the UK Brain Bank criteria for PD diagnosis [11]. After the collection of CSF samples by lumbar puncture, the samples were centrifuged for 10 minutes at 1,500 $\mathrm{xg}$, aliquoted, and stored at $-80^{\circ} \mathrm{C}$ within one hour of acquisition. The demographic and clinical characteristics of the PD patients and control individuals are shown in Supplemental Table S1.

\section{Sample preparation of cerebrospinal fluids for mass spectrometry analysis}

For quantification of pY39 a-syn peptides from PD patients and control samples, $20 \mathrm{fmol}$ of synthetic heavy pY39 a-syn peptide was added to CSF. Cerebrospinal fluid proteins were lysed in $4 \mathrm{M}$ urea and 50 $\mathrm{mM}$ triethylammonium bicarbonate (TEAB) followed by a reduction with $10 \mathrm{mM}$ dithiothreitol for $1 \mathrm{~h}$ at RT and alkylation with $30 \mathrm{mM}$ iodoacetamide for $30 \mathrm{~min}$ at RT in the dark. The proteins were then digested with an endoproteinase Lys-C (1:100; Wako Chemicals, Richmond, VA) by incubating at room temperature for three hours. Sequentially trypsin digestion was conducted by diluting the urea concentration to $2 \mathrm{M}$ by adding 1 volume of $50 \mathrm{mM}$ TEAB followed by adding sequencing-grade trypsin (1:50; Promega, Madison, $\mathrm{WI}$ ) and incubating at $37^{\circ} \mathrm{C}$ overnight. The peptide samples were desalted with $\mathrm{C}_{18}$ Sep-Pak (Waters Corporation, Milford, MA) and freeze-dried.

\section{Enrichment of pY39 a-syn peptide}

The pY39 a-syn peptides (heavy K8 $\left({ }^{13} \mathrm{C}_{6} /{ }^{15} \mathrm{~N}_{2}\right)$ [EGVLYVGSK*] and endogenous [EGVLYVGSK]) were enriched by performing phosphotyrosine peptide enrichment with PTMScan pY1000 antibody according to manufacturer's instruction with minor modifications (Cell Signaling Technology, Danvers, MA). Briefly, the CSF peptides derived from $1 \mathrm{ml}$ of CSF were reconstituted in $200 \mu \mathrm{l}$ of immunoaffinity purification buffer ( $50 \mathrm{mM}$ MOPS, pH 7.2, $10 \mathrm{mM} \mathrm{Na}_{2} \mathrm{HPO}_{4}$ and $50 \mathrm{mM} \mathrm{NaCl}$ ). The peptide solution was cleared by centrifugation for $5 \mathrm{~min}$ at $10,000 \mathrm{xg}$ at $4^{\circ} \mathrm{C}$ and the supernatant was subject to the phosphotyrosine 
enrichment. After washing $20 \mu \mathrm{l}$ of phosphotyrosine agarose beads three times with PBS, the CSF peptide solution was added to the washed beads followed by incubation at $4^{\circ} \mathrm{C}$ for $2 \mathrm{~h}$ with rotation.

Subsequently, the supernatant was removed and the beads were washed once with ice-cold water. The bound tyrosine-phosphorylated peptides were eluted by adding $55 \mu \mathrm{l}$ of $0.15 \%$ trifluoroacetic acid (TFA) and incubated at RT for $10 \mathrm{~min}$. After incubation, the tube was centrifuged at 2,000 x g for $1 \mathrm{~min}$ and the solution was transferred to a new tube. This elution was repeated once again with $50 \mu 10.15 \% \mathrm{TFA}$. The eluate was dried using a SpeedVac and the phosphorylated peptides were enriched again using $\mathrm{TiO}_{2}$ beads as described previously [12]. Briefly, $0.6 \mathrm{mg}$ of $\mathrm{TiO}_{2}$ beads ( $\mathrm{Titansphere)} \mathrm{resuspended} \mathrm{in} 40 \mu \mathrm{l}$ of binding buffer ( $65 \%$ acetonitrile (ACN) and $2 \%$ TFA) were added to the peptides followed by incubation at RT for 20 min with shaking at 1,400 rpm. The peptides were transferred to a $C_{8}$ StageTip and centrifuged at 2,000 $\mathrm{xg}$ for $2 \mathrm{~min}$. Two hundred $\mu \mathrm{l}$ of the washing buffer ( $65 \% \mathrm{ACN}$ and $0.1 \% \mathrm{TFA}$ ) was added and centrifuged at 2,000 $\mathrm{xg}$ for $5 \mathrm{~min}$. This washing was repeated once again. The phosphopeptides were eluted by adding $40 \mu \mathrm{l}$ of elution buffer $\left(1 \% \mathrm{NH}_{4} \mathrm{OH}\right.$ and $\left.40 \% \mathrm{ACN}\right)$ and centrifuging at $200 \mathrm{xg}$ for 2 min. The eluted peptides were then dried using a SpeedVac followed by reconstitution in $15 \mu \mathrm{l}$ of $0.1 \%$ formic acid prior to mass spectrometry analysis.

\section{Calculation of the limits of detection and quantification}

The limits of detection (LOD) were calculated as: $L O D=\mu_{B}+t_{(1-\beta)}\left(\sigma_{B}+\sigma_{S}\right) / \sqrt{ } n$, where $\mu_{B}$ is the estimated mean of blank samples, $t_{(1-\beta)}$ is 95 percentile of the standard $t$ distribution on $f$ degrees of freedom, $\sigma_{B}$ is the standard deviation of the blank samples, $\sigma_{S}$ is the standard deviation of the low concentration samples, and $\mathrm{n}$ is the number of replicates. The limits of quantification (LOQ) were estimated as $3 x$ LOD [13].

\section{Detection of total alpha-synuclein}

To normalize the amount of pY39 a-syn peptide in each sample based on the total a-syn present in each sample, the amount of total a-syn in each sample was also measured. Twenty fmol of $\mathrm{K} 8\left({ }^{13} \mathrm{C}_{6} /{ }^{15} \mathrm{~N}_{2}\right)$ heavy a-syn peptide (EGVLYVGSK*) for the quantification of the endogenous a-syn peptide were added to $5 \mu \mathrm{g}$ of CSF peptides followed by desalting with $\mathrm{C}_{18}$ StageTip and LC-MS/MS analysis.

\section{LC-MS/MS analysis}

The fractionated peptides were analyzed on an Orbitrap Fusion Lumos Tribrid Mass Spectrometer coupled to an EASY-nLC 1200 nano-flow liquid chromatography system (Thermo Fisher Scientific). The peptides from each fraction were reconstituted in $15 \mu \mathrm{l}$ of $0.1 \%$ formic acid and loaded onto an Acclaim 
PepMap100 Nano-Trap Column $\left(100 \mu \mathrm{m} \times 2 \mathrm{~cm}\right.$, Thermo Fisher Scientific) packed with $5 \mu \mathrm{m} \mathrm{C}_{18}$ particles at a flow rate of $5 \mu \mathrm{l}$ per min. The flow rate employed was $250 \mathrm{nl} / \mathrm{min}$ using a linear gradient of $10 \%$ to $35 \%$ solvent B ( $0.1 \%$ formic acid in $95 \%$ acetonitrile) over 45 minutes on an EASY-Spray column ( $50 \mathrm{~cm} \mathrm{x}$ $75 \mu \mathrm{m}$ ID, Thermo Fisher Scientific) packed $2 \mu \mathrm{m} \mathrm{C}_{18}$ particles (Thermo Fisher Scientific), which was fitted with an EASY-Spray ion source operated at a voltage of $2.7 \mathrm{kV}$. Mass spectrometry analysis was completed in a data-dependent manner with a full scan in the mass-to-charge ratio $(\mathrm{m} / \mathrm{z})$ range of 350 to 1,550 followed by targeted MS2. MS1 was measured at a resolution of 120,000 (at $m / z$ of 200 ). MS2 scan was acquired by fragmenting precursor ions using the higher-energy collisional dissociation (HCD) method and detected at a mass resolution of 30,000 (at $\mathrm{m} / \mathrm{z}$ of 200 ). Automatic gain control was set to 500,000 and 100,000 ions for MS1 and MS2, respectively. The maximum ion injection time for MS1 was set to 100 milliseconds. Maximum ion times for MS2 were set to 2,500 and 500 milliseconds for pY39 asyn and a-syn, respectively. HCD normalized collisional energy (NCE) was set to 25 , if not specified. The precursor isolation window was set to $1.6 \mathrm{~m} / \mathrm{z}$. Internal calibration was carried out using the lock mass option ( $m / z 445.1200025)$ from ambient air. For the light and heavy pY39 a-syn peptides, $m / z 516.244$ and $m / z 520.251$ were monitored, respectively. For the light and the heavy Y39 a-syn peptides, $m / z$ 476.261 and $\mathrm{m} / \mathrm{z} 480.268$ were monitored, respectively.

\section{Data analysis}

The quantification of relative peptide abundance was performed using Skyline software [14]. The levels of pY39 a-syn were normalized by total alpha-synuclein.

\section{Results}

To detect pY39 a-syn peptide in CSF samples, we initially tried to detect it directly from the digests of CSF samples using PRM but were not able to detect it. Subsequently, we tried an enrichment using an antibody against a-syn protein or enrichment of phosphopeptides using $\mathrm{TiO}_{2}$ or Immobilized metal affinity chromatography (IMAC). However, none of these enrichment methods were successful in detecting pY39 a-syn peptide. We reasoned that because pY39 a-syn is a tyrosine-phosphorylated peptide, we could further reduce peptide complexity by performing phosphotyrosine peptide enrichment to remove phosphoserine and phosphothreonine peptides, which constitute the majority of phosphopeptides. Using this procedure, we were able to detect the endogenous pY39 a-syn peptide from $9 \mathrm{ml}$ of CSF (Figure 1A and B). Because obtaining $9 \mathrm{ml}$ of CSF is not practical in most cases for biomarker detection, we decided to further optimize our methods to reduce the amount of CSF required for the analysis.

\section{Optimizing the detection of pY39 a-syn peptide}


To improve our detection sensitivity, we systematically evaluated the effect of HCD NCE energy, ion transfer capillary temperature and ESI spray voltage on detection. For the HCD NCE evaluation, the HCD NCE value was increased from 24 to 32 . The pY39 a-syn peptide showed the highest intensity at 25 of HCD NCE (Figure 2A). Next, we evaluated ion transfer capillary temperature increasing from 160 to 400 and pY39 a-syn peptide showed the highest intensity at $180^{\circ} \mathrm{C}$ (Figure 2B). We then evaluated ESI voltage optimization by increasing from 1,500 to 3,000. pY39 a-syn peptide showed the highest intensity at 3,000 V (Figure 2C). Interestingly, the intensity at 3,000 V was $>3.5$-fold compared to the one observed at $1,500 \mathrm{~V}$. Although the HCD NCE and the ion transfer capillary temperature did not change the intensity of pY39 a-syn greatly, the ESI voltage seemed to be critical in increasing the sensitivity. We applied the optimized parameters for the detection of pY39 a-syn except that we opted to use 2,700 V instead of $3,000 \mathrm{~V}$ for preserving column stability. A calibration curve with these optimized parameters permitted us to detect the target peptide at sub-attomole levels. The LOD and LOQ were 0.44 and 1.32 attomoles, respectively. The coefficient of variations (CV) was calculated as $26.19 \%$ at 1 attomole, $4.45 \%$ at 10 attomoles, $4.84 \%$ at 100 attomoles, $10.03 \%$ at 1 femtomole, $4.45 \%$ at 10 femtomoles and $8.93 \%$ at 100 femtomoles. The average CV of the 6 concentrations was $9.81 \%$. (Supplemental Figure S1).

\section{Development of an enrichment method for pY39 peptides}

To detect the pY39 a-syn peptide from an even smaller volume of CSF, we optimized the enrichment method as well. Even after the enrichment of phosphotyrosine peptides, $80-90 \%$ of peptides are still nonphosphorylated ones and they interfere with the detection of target peptides. Thus, the second step of $\mathrm{TiO}_{2}$-based phosphopeptide enrichment should further remove the non-phosphorylated peptides, and thereby, it will increase the sensitivity of detecting target peptides (Figure 3A). Thus, we developed a twostep enrichment method by combining the antibody-based phosphotyrosine peptide enrichment method with the $\mathrm{TiO}_{2}$-based phosphopeptide enrichment method. To optimize this two-step detection method, we first evaluated the ratio of phosphotyrosine antibody-conjugated agarose beads to the input peptide amount. One femtomole of heavy pY39 a-syn peptide was incubated with various volumes of antibodyconjugated beads ranging from 2.5 ul to 80 ul. The relative intensity of the heavy pY39 a-syn peptide showed saturation at $20 \mu \mathrm{l}$ of the agarose beads (Figure 3B). We next optimized the ratio of $\mathrm{TiO}_{2}$ beads to input peptide amount. For this, 2 femtomoles of heavy pY39 a-syn peptide was incubated with various amounts of $\mathrm{TiO}_{2}$ beads ranging from $0.1 \mathrm{mg}$ to $3.2 \mathrm{mg}$. The pY39 a-syn showed the highest intensity at $0.8 \mathrm{mg}$ of $\mathrm{TiO}_{2}$ beads (Figure 3C).

\section{Measurement of pY39 a-syn peptides in CSF samples from PD and control individuals}

Since the goal of this study was to quantify the endogenous pY39 a-syn in CSF samples from PD patients and control individuals, we applied the optimized mass spectrometry parameters and sample 
preparation procedure to detect the pY39 a-syn peptides from 4 PD and 4 control individuals as shown in Figure 4A. To minimize any experimental bias and maximize the accuracy of quantification, synthetic heavy pY39 a-syn peptide was added to all CSF samples at the beginning of the sample preparation step [15]. When pY39 a-syn levels in $1 \mathrm{ml}$ of CSF samples from PD patients were compared to the one from controls, there was no statistically significant difference (Figure 4B and Supplemental Table S2). Because it is already known that total a-syn levels are decreased in PD patients, we postulated that the levels of pY39 a-syn normalized to total Y39 a-syn levels might help distinguish PD patients from controls. For this, we first measured the total a-syn levels by spiking a synthetic heavy a-syn peptide (Supplemental Figure S2). As we expected, a-syn in CSF from PD patients showed a decreased abundance (Figure 4C). Most importantly, the ratio of pY39 a-syn to total a-syn in CSF from PD patients showed a noticeable increase $(2.5$ fold $)$ with statistical significance $\left(P\right.$ value $\left.=8.4 \times 10^{-5}\right)$ (Figure $\left.4 \mathrm{D}\right)$. These results are potentially exciting and will have to be validated in a larger cohort.

\section{Discussion}

In this study, we developed a two-step enrichment method to detect endogenous pY39 a-syn peptide in a sensitive manner from CSF samples using the PRM method. This approach enabled us to detect the phosphorylated target peptide present at attomole levels per ml of CSF. The enrichment efficiency of phosphotyrosine peptides using the anti-phosphotyrosine antibody-conjugated beads in the first enrichment is usually $<15 \%$ owing to non-specifically bound peptides even after 5 washes. These nonspecifically bound peptides increase the noise in PRM experiments thereby reducing the detection sensitivity. These non-specifically bound peptides can be removed by more extensive washing but it will result in the loss of the target peptide as well. For this reason, instead of washing the beads stringently losing the target peptide, we chose to remove the non-specifically bound peptides by a second enrichment step using TiO2 beads. Using this strategy, we were able to improve the detection sensitivity of the target peptide to attomole/ml levels. While the endogenous pY39 a-syn peptide levels alone did not show a statistically significant difference between PD and control CSFs, the relative abundance of $\mathrm{pY} 39 /$ total asyn was strikingly different between the two groups with statistical significance. Our results suggest that the stoichiometry of tyrosine phosphorylation of a-syn might be increased in patients with PD. The development of this method now makes it possible to test the utility of the pY39/total a-syn ratio as a potential readout for c-Abl activity as well. In addition, this method is broadly applicable to the detection of other phosphotyrosine peptides in biological samples with minor modifications.

\section{List Of Abbreviations}

PD: Parkinson's disease

a-syn: a-synuclein

pY39 a-syn: phosphorylation of the tyrosine residue at position 39 of a-syn 
$\mathrm{TiO}_{2}$ : titanium oxide

PTMs: post-translational modifications

PRM: parallel reaction monitoring

NPH: normal pressure hydrocephalus

\section{Declarations}

\section{Ethics approval and consent to participate}

This study was approved by the Johns Hopkins University School of Medicine Institutional Review Board. Informed consent was obtained from all study participants

\section{Consent for publication}

Not applicable

\section{Availability of data and materials}

All mass spectrometry data and search results have been deposited in the ProteomeXchange Consortium via the PRIDE partner repository with the dataset identifier PXD012202 and project name 'Development of a method for the quantification of tyrosine 39 phosphorylated alpha-synuclein in human cerebrospinal fluid' [16]. Reviewers can access the dataset by using 'reviewer43678@ebi.ac.uk' as ID and 'jnapGY1k' as a password.

\section{Competing interests}

We have no conflict of interest to declare.

\section{Authors' contributions}

C.H.N., T.M.D., and A.P. designed research; L.S.R. and A. R. M. collected CSF; C. H. N. and G. S. performed mass spectrometry analysis. C.H.N, G.S., L.S.R, A. R. M., V. L. D., T. M. D. and A.P wrote the manuscript. C. H. N., T. M. D. and A.P. supervised research.

\section{Funding}

This work was supported by NIH grants NINDS, P50NS38377 and U01NS082133 U01NS097049 and the Wellcome Trust/DBT India Alliance Margdarshi Fellowship (grant number IA/M/15/1/502023 to A.P.).

\section{Acknowledgments}


The authors acknowledge the joint participation by the Diana Helis Henry Medical Research Foundation through its direct engagement in the continuous active conduct of medical research in conjunction with The Johns Hopkins Hospital and the Johns Hopkins University School of Medicine and the Foundation's Parkinson's Disease Program H-2013. T.M.D. is the Leonard and Madlyn Abramson Professor in Neurodegenerative Diseases. We also acknowledge the support of the shared instrumentation grant (S100D021844).

\section{References}

1. Brahmachari, S., et al., c-Abl and Parkinson's Disease: Mechanisms and Therapeutic Potential. J Parkinsons Dis, 2017. 7(4): p. 589-601.

2. Oueslati, A., Implication of Alpha-Synuclein Phosphorylation at S129 in Synucleinopathies: What Have We Learned in the Last Decade? Journal of Parkinsons Disease, 2016. 6(1): p. 39-51.

3. Brahmachari, S., et al., Activation of tyrosine kinase c-Abl contributes to alpha-synuclein-induced neurodegeneration. J Clin Invest, 2016. 126(8): p. 2970-88.

4. Burmann, B.M., et al., Regulation of alpha-synuclein by chaperones in mammalian cells. Nature, 2019.

5. Jimenez-Jimenez, F.J., et al., Cerebrospinal fluid biochemical studies in patients with Parkinson's disease: toward a potential search for biomarkers for this disease. Front Cell Neurosci, 2014. 8: p. 369.

6. Bourmaud, A., S. Gallien, and B. Domon, Parallel reaction monitoring using quadrupole-Orbitrap mass spectrometer: Principle and applications. Proteomics, 2016. 16(15-16): p. 2146-2159.

7. Brinkmalm, G., et al., A Parallel Reaction Monitoring Mass Spectrometric Method for Analysis of Potential CSF Biomarkers for Alzheimer's Disease. Proteomics Clinical Applications, 2018. 12(1).

8. Preisinger, C., et al., Imatinib-dependent tyrosine phosphorylation profiling of Bcr-Abl-positive chronic myeloid leukemia cells. Leukemia, 2013. 27(3): p. 743-746.

9. Ciccimaro, E., S.K. Hanks, and I.A. Blair, Quantification of Focal Adhesion Kinase Activation Loop Phosphorylation as a Biomarker of Src Activity. Molecular Pharmacology, 2009. 75(3): p. 658-666.

10. Razavi, M., et al., High precision quantification of human plasma proteins using the automated SISCAPA Immuno-MS workflow. New Biotechnology, 2016. 33(5): p. 494-502.

11. Hughes, A.J., et al., Accuracy of clinical diagnosis of idiopathic Parkinson's disease: a clinicopathological study of 100 cases. J Neurol Neurosurg Psychiatry, 1992. 55(3): p. 181-4.

12. Tan, H., et al., Refined phosphopeptide enrichment by phosphate additive and the analysis of human brain phosphoproteome. Proteomics, 2015. 15(2-3): p. 500-7.

13. Addona, T.A., et al., Multi-site assessment of the precision and reproducibility of multiple reaction monitoring-based measurements of proteins in plasma. Nat Biotechnol, 2009. 27(7): p. 633-41.

14. MacLean, B., et al., Skyline: an open source document editor for creating and analyzing targeted proteomics experiments. Bioinformatics, 2010. 26(7): p. 966-968. 
15. Shuford, C.M., et al., Peptide production and decay rates affect the quantitative accuracy of protein cleavage isotope dilution mass spectrometry (PC-IDMS). Mol Cell Proteomics, 2012. 11(9): p. 814-23.

16. Vizcaino, J.A., et al., 2016 update of the PRIDE database and its related tools. Nucleic Acids Res, 2016. 44(D1): p. D447-56.

\section{Supplemental Figure Legends}

Supplemental Table S1. The demographic and clinical characteristics of PD patients and control individuals.

Supplemental Table S2. Quantification results for total a-syn and pY39 a-syn in the CSF of PD patients and control individuals.

Supplemental Figure S1. Calibration curve of the heavy synthetic pY39 a-syn peptide in the presence of $10 \mathrm{ng}$ or $2 \mu \mathrm{g}$ of CSF peptides.

Supplemental Figure S2. Experimental strategy for the quantification of the total a-syn in CSF.

\section{Figures}




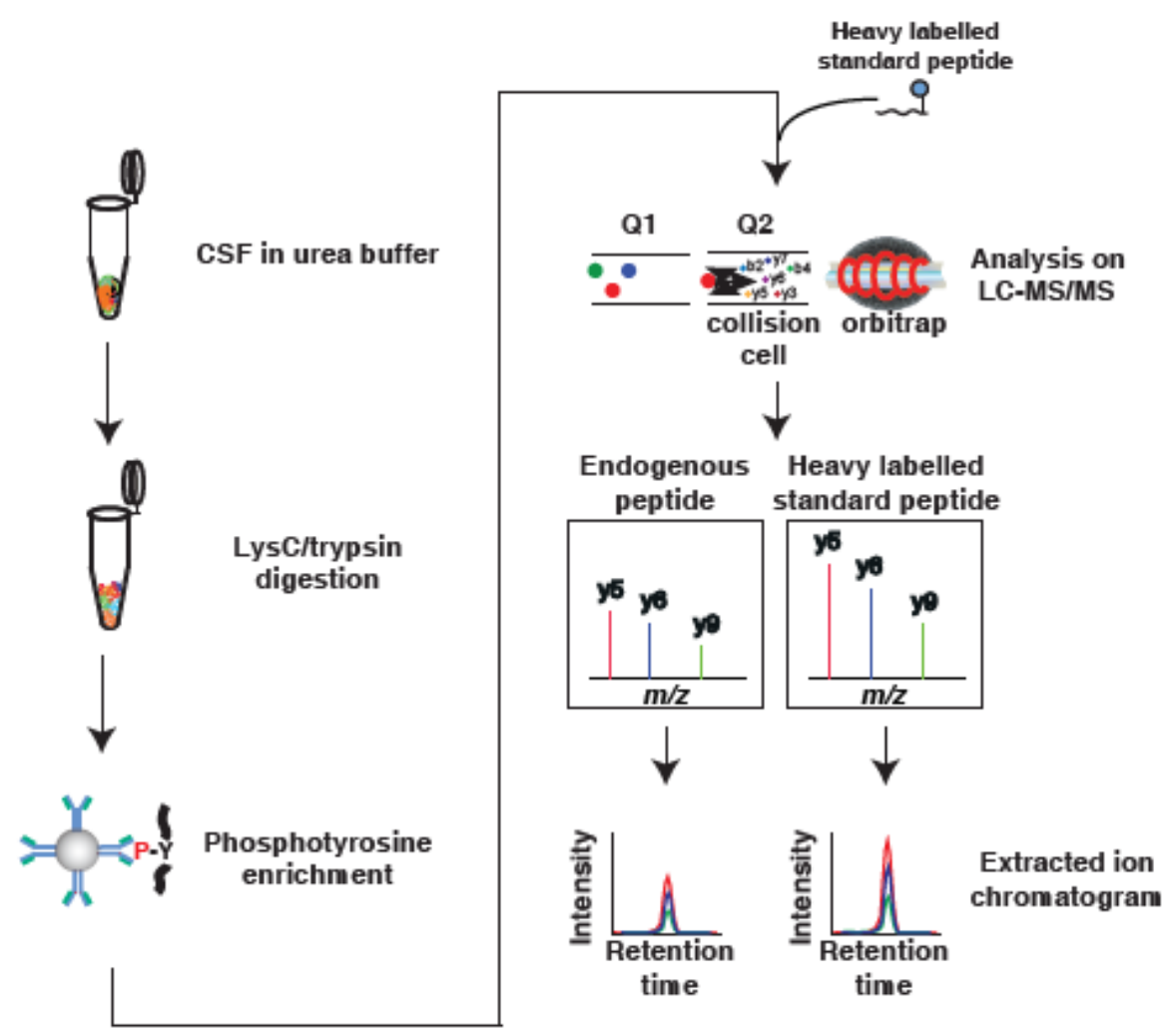

B

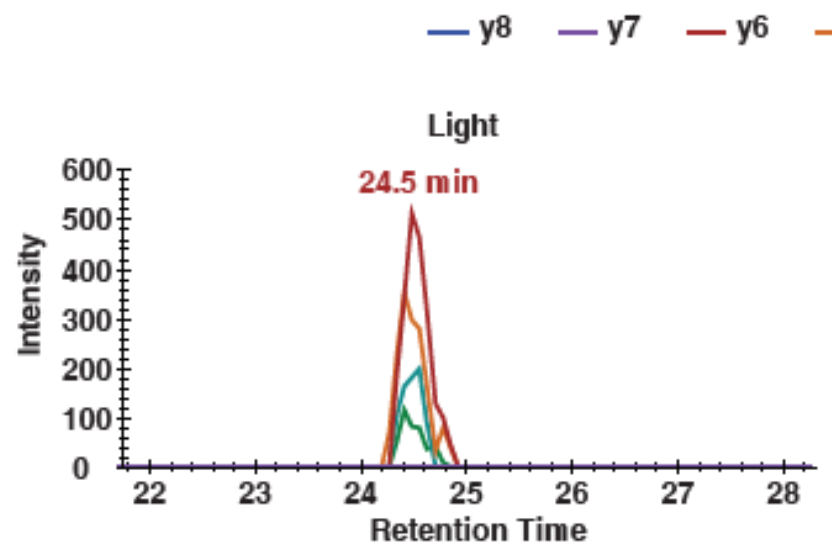

$\begin{array}{lll}\mathrm{y} & -\mathrm{y} 4 & -\mathrm{y} 3\end{array}$

\section{Figure 1}

he schematic diagram for the research strategy and the detection of endogenous pY39 『-syn peptides. A. The experimental strategy for pY39 \-syn enrichment using an anti-phosphotyrosine antibody. CSF proteins were digested with Lys-C and trypsin followed by phosphotyrosine peptide enrichment. To validate the detection of the endogenous pY39 『-syn, a heavy pY39 『-syn standard peptide was added before PRM analysis. The endogenous light and heavy standard pY39 『-syn were monitored under PRM 
mode followed by a quantification using Skyline software. B. The extracted chromatogram of y ion series from either endogenous or heavy standard pY39 \-syn.

A

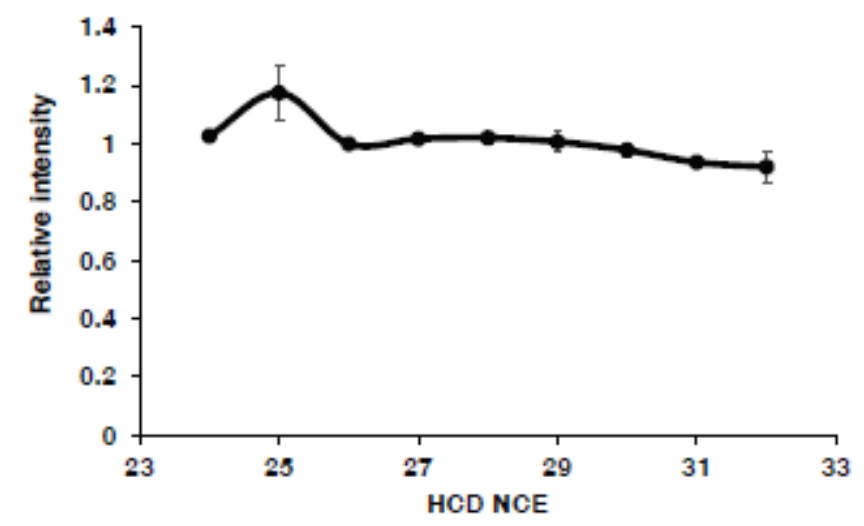

C

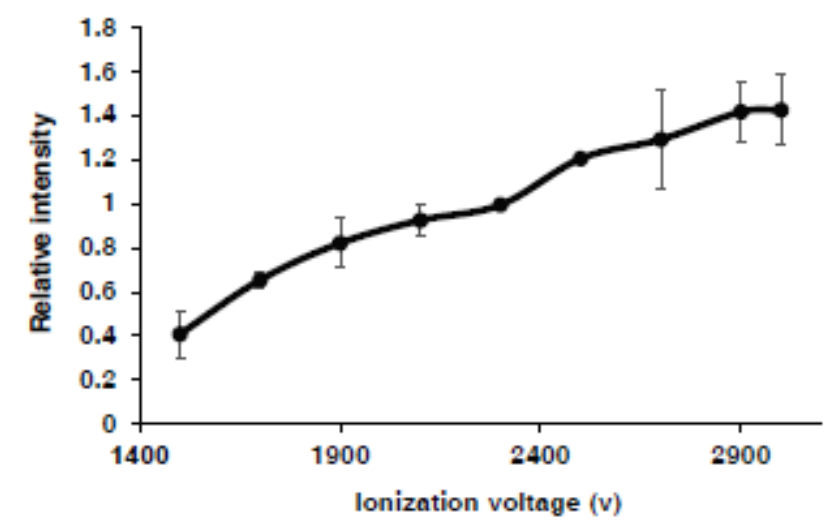

B

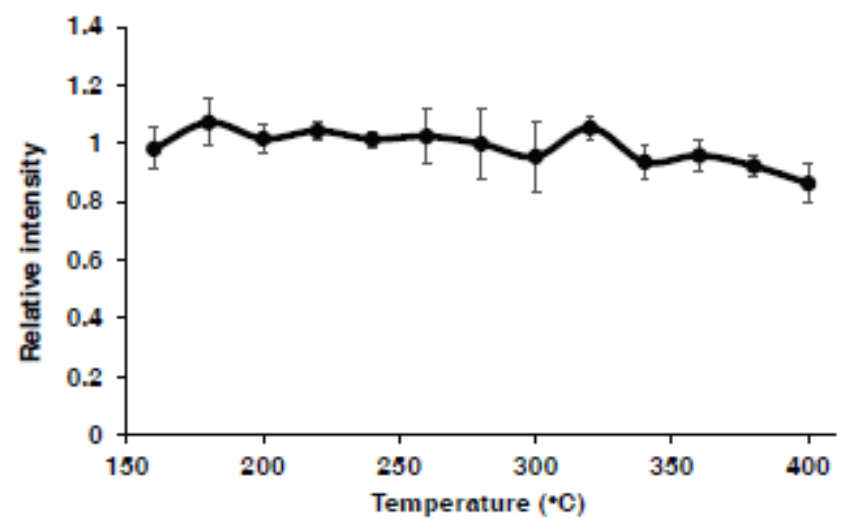

\section{Figure 2}

Optimization of mass spectrometry parameters for pY39 peptide detection A. The relative intensity of the standard pY39 \-syn peptides with different HCD NCEs. B. The relative intensity of the standard pY39 \syn peptides with different ion transfer tube capillary temperatures. C. The relative intensity of the standard pY39 『-syn peptides with different ESI voltages. 
A

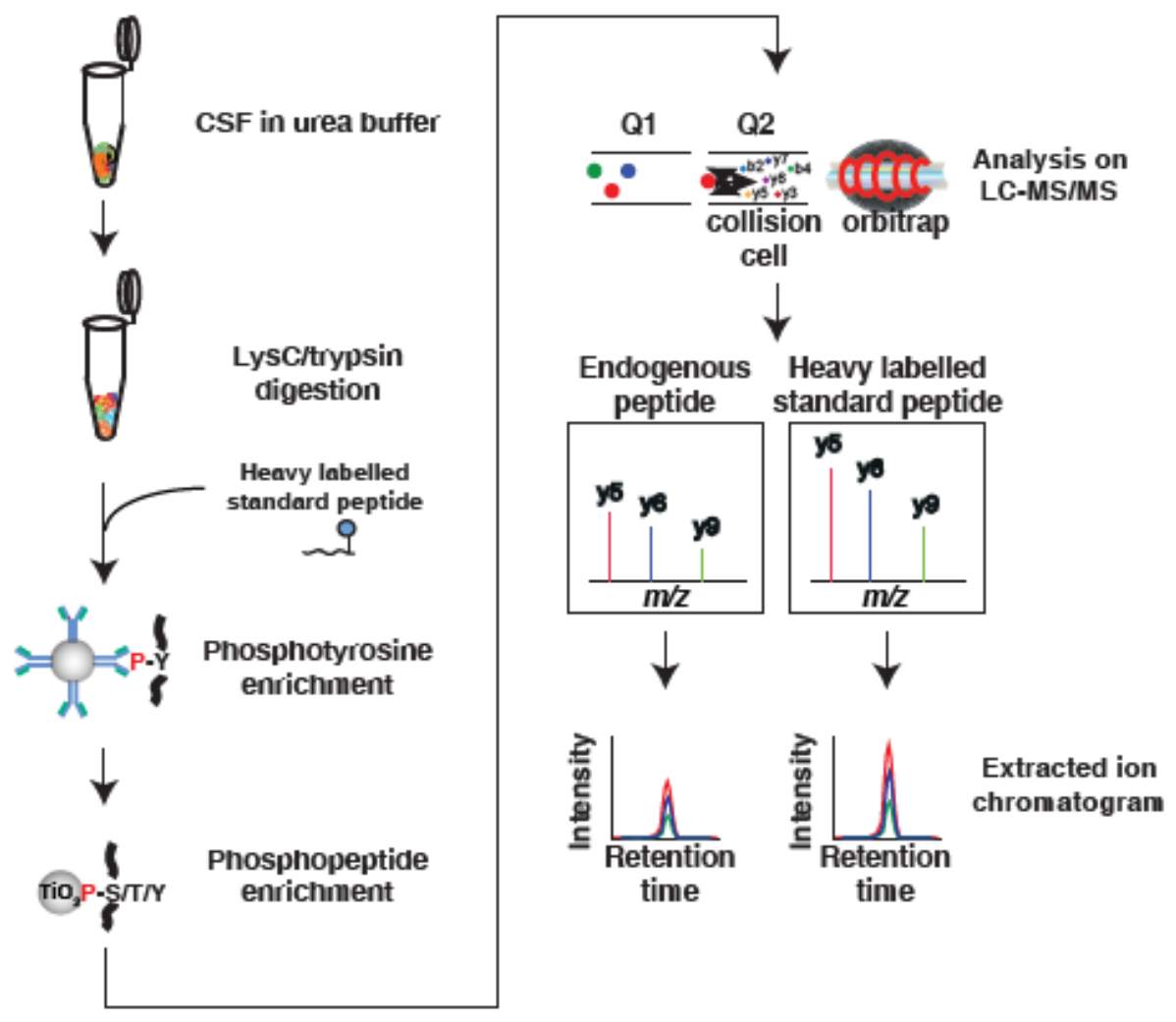

B
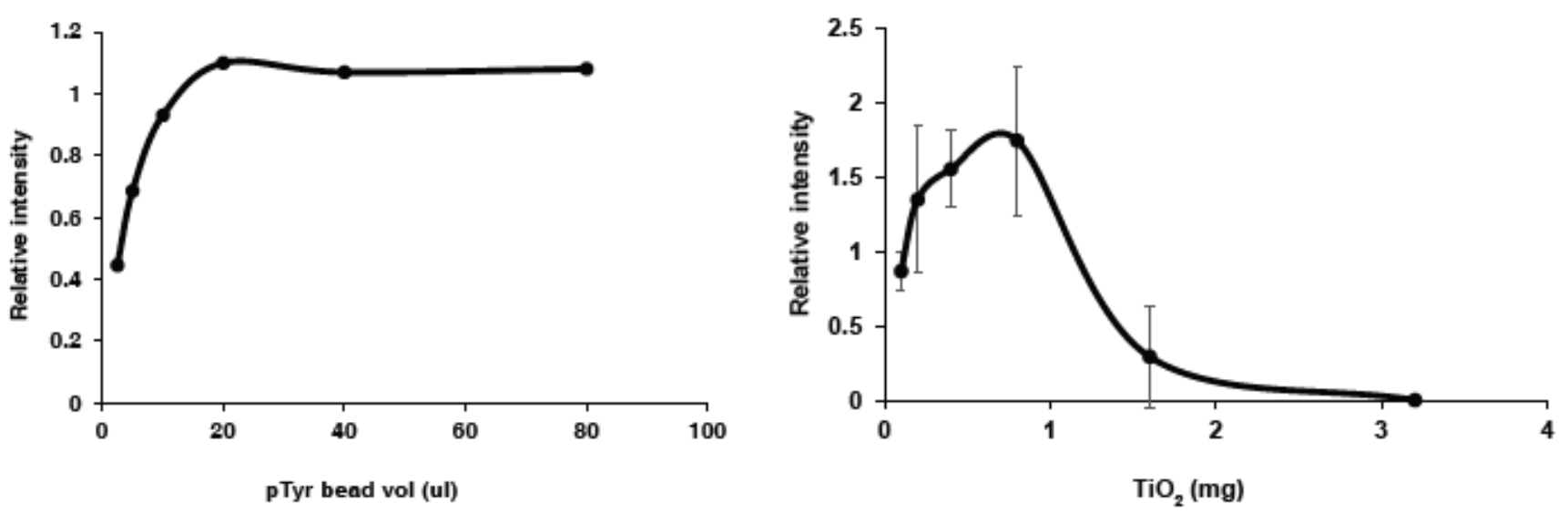

\section{Figure 3}

Two-step enrichment strategy and the optimization of the two-step enrichment experiment conditions A. The experimental strategy for pY39 『-syn enrichment using the two-step enrichment approach in which phosphotyrosine peptides were enriched followed by total phosphopeptides were enriched using TiO2 beads. CSF proteins were digested with Lys-C and trypsin. To monitor the enrichment efficiency, heavy standard pY39 \-syn peptide was added before the two-step enrichment and the enrichments were 
conducted. The endogenous light and heavy standard pY39 $\square$ Syn were monitored under PRM mode followed by quantification using Skyline software. B. Different volumes of agarose beads coupled with anti-phosphotyrosine antibodies were incubated with the target peptides to investigate the best ratio of anti-phosphotyrosine agarose beads to the target peptides. C. Different amounts of TiO2 beads were incubated with the target peptides to investigate the best ratio of $\mathrm{TiO} 2$ beads to the target peptides.

A
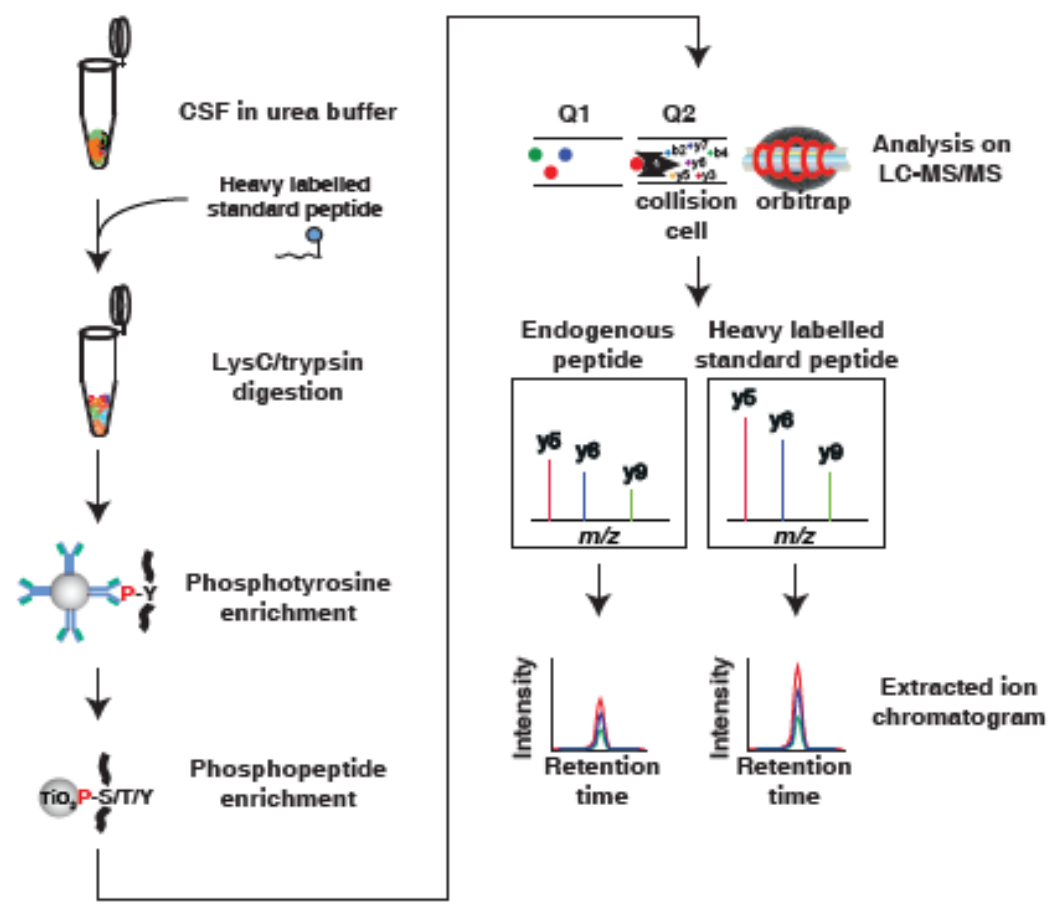

B

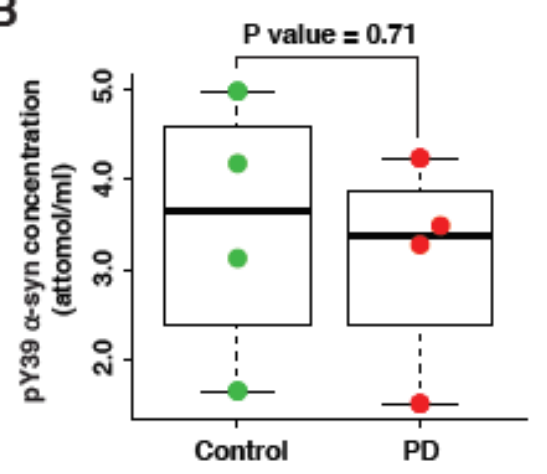

C

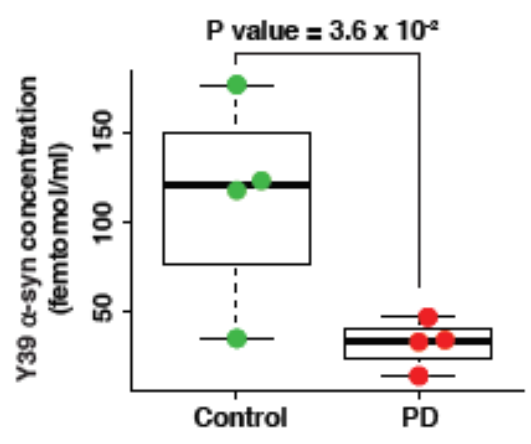

D

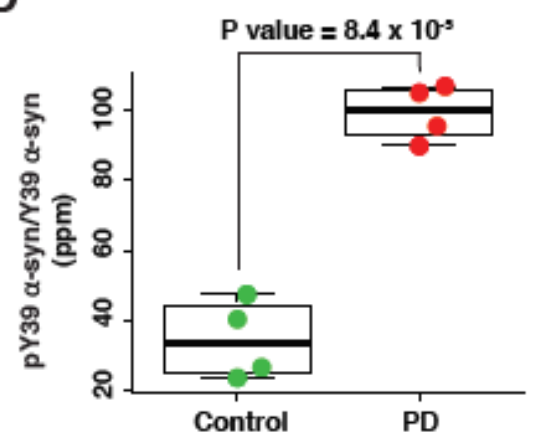

Figure 4 
Quantification of pY39 『-syn peptide in CSF samples from PD and control individuals A. The experimental

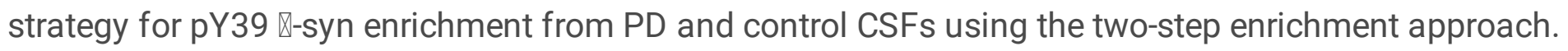
CSF proteins were digested with Lys-C and trypsin followed by the two-step enrichment. To minimize experimental biases, heavy standard pY39 $₫$-syn peptide was added before starting the experiment. The

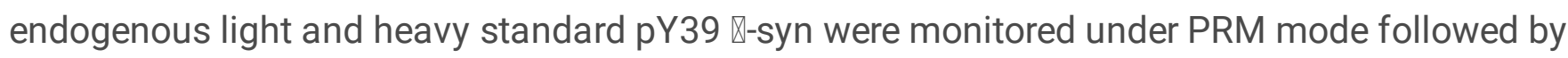
quantification using Skyline software. B. The abundances of pY39 『-syn in PD and control CSFs. C. The

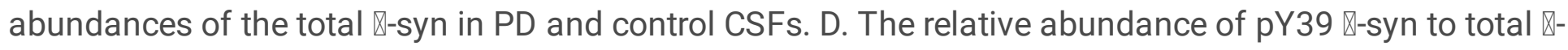
syn in PD and control CSFs.

\section{Supplementary Files}

This is a list of supplementary files associated with this preprint. Click to download.

- SupplementalFigure2.jpg

- SupplementalTableS2v04.xlsx

- SupplementalDataS1.sky.view

- SupplementalDataS1.sky

- SupplementalFigure1.jpg

- SupplementalDataS1.skyd

- SupplementalTableS1v01.xlsx 